\title{
Atividades científicas e tecnológicas desenvolvidas com Arecaceae
}

\section{Roseli Aparecida Cardoso Vieira e Medeiros ${ }^{1}$, Adelson Antônio de Castro ${ }^{2}$, Murilo Malveira Brandão ${ }^{1}$ e Dario Alves de Oliveira ${ }^{1}$,*}

${ }^{1}$ Programa de Pós-Graduação Profissional em Biotecnologia. Universidade Estadual de Montes Claros (Unimontes). Campus Universitário Prof. Darcy Ribeiro. Av. Prof. Rui Braga, S/No. Vila Mauriceia. Montes Claros-MG, Brasil (CEP 39401-089).

${ }^{2}$ Universidade Federal do Rio de Janeiro. Av. Pedro Calmon, 900. Cidade Universitária. Rio de Janeiro-RJ, Brasil (CEP 21941-908). *E-mail: dario.oliveira@unimontes.br.

Resumo. Espécies de palmeiras (Arecaceae) são fontes de matérias-primas renováveis para atender às demandas das indústrias de alimentos, das farmacêuticas, de biocombustíveis, de cosméticos e outras. 0 presente trabalho teve como objetivo identificar a produção de conhecimento científico e tecnológico com o uso das palmeiras Mauritia flexuosa Lin. Fil. (buriti), Acrocomia aculeata (Jacq.) Lodd. ex Mart. (macaúba) e Syagrus oleracea (Mart.) Becc. (guariroba), que são utilizadas para desenvolvimento de produtos em diferentes áreas. Foram realizadas pesquisas exploratórias no banco de dados da Web of Science e a proteção de tecnologias em bancos de dados de patentes do Instituto Nacional de Propriedade Industrial (INPI), Escritório Europeu de Patentes (ESPACENET) e Escritório Latino-Americano de Patentes (LATIPAT). Os resultados permitiram identificar 223 publicações de artigos com buriti e 33 depósitos de patentes, para a macaúba, foram identificadas 192 publicações e 20 depósitos de patentes, e para a guariroba, 20 publicações de artigos e dois depósitos de patentes. Foi observado que empresas internacionais sobressaíram em inovação com o buriti, pois realizaram nove do total de 11 depósitos na área de cosmetologia. o Brasil destaca-se como o maior gerador de conhecimento científico relacionado às Arecaceae, com publicações voltadas para as áreas da agricultura, botânica e ciências dos alimentos. Entretanto, os resultados demonstraram que os depósitos de patentes realizados por brasileiros de empresas privadas, instituições científicas e tecnológicas e inventores independentes foram pequenos. 0 distanciamento entre a produção de conhecimento e o desenvolvimento de produtos indica que o Brasil é incipiente em ofertar inovações para o mercado com a utilização de matérias-primas das palmeiras estudadas.
Recebido

19/06/2020

Aceito

$30 / 10 / 2020$

Disponível on line

$02 / 11 / 2020$

Publicado

$31 / 12 / 2020$

Acesso aberto

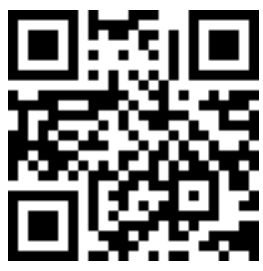

ORCID

(D) 0000-0002-5801-1533

Roseli Aparecida Cardoso Vieira e Medeiros

0000-0003-3342-2798 Adelson Antônio de Castro

ISSN 2359-1412/RBGAS-2020-0095/2020/7/17/19/1335 
Palavras-chave: Biodiversidade; Publicações científicas; Patentes; Tecnologias.

Abstract. Scientific and technological activities developed with Arecaceae. Palm tree species (Arecaceae) are sources of renewable raw materials used to meet the demands of the food, pharmaceutical, biofuel, cosmetics, and other industries. This study aimed to identify the production of scientific and technological knowledge with the use of Mauritia flexuosa Lin. Fil. (moriche palm), Acrocomia aculeata (Jacq.) Lodd. ex Mart. (grugru palm), and Syagrus oleracea (Mart.) Becc. (guariroba), which are palm species used for product development in different areas. Exploratory research was carried out in the Web of Science database and protection of technologies was researched in patent databases of the National Institute of Industrial Property (INPI), European Patent Office (ESPACENET), and Latin American Patent Office (LATIPAT). From the research, it was possible to identify 223 articles and 33 patents on moriche palm, 192 articles and 20 patents on grugru palm, and 20 articles and two patents on guariroba. It was observed that international companies stood out in innovation with the moriche palm because they filed nine of the eleven patents on cosmetics. Brazil is the largest generator of scientific knowledge related to Arecaceae species, with studies focused on agriculture, botany, and food science. However, the results showed there is a small number of patents filed by Brazilian researchers of private companies and scientific and technological institutions, as well as by independent inventors. The gap between knowledge production and product development in Brazil shows an incipient development of technologies using raw materials from the studied palm trees.

Keywords: Biodiversity; Scientific publications; Patents; Technologies.

\section{Introdução}

As palmeiras (Arecaceae) apresentam grande diversidade de espécies e são promissoras para o desenvolvimento de produtos e processos, seja com utilização do fruto in natura ou com a obtenção de extratos e óleos vegetais utilizados na indústria de alimentos, fármacos, biocombustíveis e como constituintes de formulações cosméticas (Leonardi, 2004).

A Mauritia flexuosa Lin. Fil., conhecida como buriti ou miriti, palmeira do brejo, moriche, carangucha e aguaje, produz frutos ricos em compostos bioativos com capacidade antioxidante e vitaminas A, B, C e E. Os compostos presentes possuem ferro e óleos insaturados com propriedades fotoprotetoras em função da absorção da radiação ultravioleta (Zanatta et al., 2008), que desperta interesse industrial como alternativa terapêutica e cosmética (Rosso e Mercadante, 2007). 0 óleo da polpa tem sido utilizado na produção de vermífugo, cicatrizante e energético natural, muitas vezes de acordo com o conhecimento tradicional de comunidades rurais. De modo similar, os óleos presentes nos frutos podem ser utilizados na produção do biodiesel em função da alta estabilidade oxidativa e térmica (Silva et al., 2010). 
Outra espécie de palmeira que tem grande utilidade para o desenvolvimento de produtos e processos é a Acrocomia aculeata (Jacq.) Lodd. ex Martius. É originária da América Tropical e conhecida como macaúba ou palmeira macaúba, ou, ainda, bocaiuva, macaíba, coco baboso e coco de espinho (Pires et al., 2013), sendo considerada uma das espécies mais evidentes no território brasileiro e, em virtude de sua fácil adaptação a diferentes ecossistemas, é apontada como economicamente promissoras (Motta et al., 2002). A produtividade de óleo da espécie tem despertado interesse socioeconômico (Roscoe et al., 2007), em função da rentabilidade de aproximadamente 4.500 litros por ha/ano, volume maior do que os produzidos com culturas oleaginosas estabelecidas como a soja, com 450 litros de óleo ha/ano e o arroz com 770 litros de óleo por ha/ano (Carvalho et al., 2011). 0 rendimento de óleo da macaúba é menor que o óleo da espécie Elaeis guineensis (dendê) que produz de 4 a 6 toneladas por hectare (Barcelos et al., 1999), todavia, tem potencial equivalente de utilização na indústria de resinas e lubrificantes (Bhering, 2018) e na crescente industrialização sustentável em biorrefinarias (Nunes, 2013). Os frutos da macaúba demonstram relevância para a indústria farmacêutica, alimentícia e cosmética na fabricação de hidratantes, sabonetes e xampus (Siqueira, 2012). A polpa de macaúba pode ser usada em diferentes produtos alimentares devido ao valor nutricional e energético além da presença de compostos bioativos como tocoferóis, carotenoides, compostos fenólicos e potenciais antioxidantes. A amêndoa possui concentração de ácidos graxos que possibilita a produção de óleo comestível, óleo para lamparina, detergente, sabão, biodiesel, hidratante capilar, laxante (Oliveira, 2014).

A Syagrus oleracea (Mart.) Becc., outra palmeira nativa, possui diversas denominações populares tais como guariroba, gueroba, gariroba, gairoba, palmito amargoso, catolé, coco babão, pati amargoso, coco amargoso, coqueiro amargoso. É uma espécie relevante no contexto do desenvolvimento regional devido à produção do peculiar palmito amargo, óleos ricos em lipídeos e antioxidantes naturais que podem ser usados em cosméticos (Dias, 2014). Podem também serem utilizados para retardar o envelhecimento cutâneo e recuperar as funções dos constituintes químicos que compõe o extrato córneo (Abreu, 1997). 0 potencial antibacteriano da Syagrus oleracea (Mart.) Becc. possibilita aplicações medicinais e, conforme o conhecimento tradicional, as flores, folhas e raízes são utilizados para o tratamento de bronquite, hemorroida e dor de coluna (Dias, 2014).

A busca e medição da produção científica permite avaliar informações tecnológicas, acompanhar o desenvolvimento das atividades científicas, por área do conhecimento, verificar quem são os autores e instituições ativas nas pesquisas e contribuir com a construção de estratégias focadas no futuro da criação de produtos e processos inovadores.

O mapeamento de tecnologias, realizado por meio de obtenção de informações em bancos de dados de patentes, permite o conhecimento dos locais em que ocorre maior competitividade técnica e a força legal e comercial de determinada tecnologia.

Os mapeamentos iniciam com a busca sistemática em bancos de dados de patentes, os quais possuem informações sobre diferentes tecnologias e permitem identificar locais de ocorrências de inovação, as tendências de locais de desenvolvimento, as diversidades de soluções técnicas para problemas do estado da arte, possíveis oportunidades de colaborações e parcerias para desenvolvimento de tecnologias, entre outros (Speziali e Sinisterra, 2015).

Neste contexto e ao considerar o potencial uso de matérias primas das palmeiras Mauritia flexuosa Lin. Fil., Acrocomia aculeata (Jacq.) Lodd. ex Mart. e Syagrus oleracea (Mart.) Becc. para o desenvolvimento de novas tecnologias, o presente trabalho buscou analisar a situação em relação às publicações científicas e aos documentos de banco de dados de patentes para identificação das principais atividades científicas e tecnológicas que tem sido desenvolvidas assim como as principais instituições envolvidas. 


\section{Material e métodos}

Foi utilizada pesquisa exploratória, com dados obtidos com a prospecção em bases nacionais e internacionais de artigos científicos e de patentes. 0 método utilizado para coleta dos dados foi a bibliometria e em seguida foram realizadas análises de conteúdos e das informações disponíveis nos bancos de dados.

\section{Bases de dados}

Foram realizadas prospecção de artigos científicos no banco de dados Web of Sience (WoS) e prospecção de produtos tecnológicos nos bancos patentários do Instituto Nacional da Propriedade Industrial (INPI), do Escritório Europeu de Patentes (ESPACENET) e do Escritório Latino Americano de Patentes (LATIPAT), com utilização de diferentes palavras chaves (Tabela 1). As expressões de buscas utilizadas para o WoS foram os nomes científicos das espécies estudadas, visto que foi observado o maior número de resultados de publicações, por meio do campo "Tópico (TS)", o qual recupera resultados no "título", "resumo" e "palavras-chave do autor". Nos bancos de dados de patentes foram utilizados os nomes científicos e os nomes populares, no campo "Busca Avançada - Palavra Chave - Resumo", por possibilitar a combinação de termos, elaboração de estratégias de buscas intercaladas com operadores lógicos booleanos "and" para pesquisar todos os termos relacionados e "or" para pesquisar qualquer um dos termos. No resumo, que faz parte do relatório descritivo utilizados no depósito de patentes, estão as principais informações relacionadas com o setor técnico ao qual pertence a invenção.

Além de ser analisado o número de patentes, foram identificados os depositantes de Instituições Científicas e Tecnológicas (ICTs) e pessoas físicas ou empresas privadas, a evolução temporal dos depósitos, os campos tecnológicos correspondentes e 0 processamento legal, para construção da situação das atividades científicas e tecnológicas desenvolvidas com as palmeiras estudadas, no período de 1999 a 2017. As buscas foram realizadas entre abril e novembro de 2017, visto que a delimitação foi feita a partir do primeiro resultado encontrado (ano da publicação-1999) e o ano da pesquisa (1999-2017).

Tabela 1. Nomes científicos e terminologias populares utilizados para prospecção de artigos científicos e produtos tecnológicos desenvolvidos com utilização das palmeiras Mauritia flexuosa Lin. Fil., Acrocomia aculeata (Jacq.) Lodd. ex Mart. e Syagrus oleracea (Mart.) Becc.

\begin{tabular}{|l|l|}
\hline Nomes científicos & \multicolumn{1}{|c|}{ Terminologias populares } \\
\hline Mauritia flexuosa Lin. Fil. & $\begin{array}{l}\text { Buriti, coqueiro buriti, buritizeiro, miriti, muriti, } \\
\text { muritim, muruti, palmeira do brejo, carandá guaçu, e } \\
\text { carandaí guaçu. }\end{array}$ \\
\hline Acrocomia aculeata (Jacq.) Lodd. ex Mart. & $\begin{array}{l}\text { Macaúba, macaíba, macaúva, boicaiuva, bocaiúva, } \\
\text { coco de catarro, coco baboso, coco de espinho, } \\
\text { macajuba, macaibeira, mucaia, mucajá e mucajaba }\end{array}$ \\
\hline Syagrus oleracea (Mart.) Becc. & $\begin{array}{l}\text { Catolé, gueiroba, guariroba, gariroba, palmito } \\
\text { amargoso, coco babão, pati amargoso, coco } \\
\text { amargoso, coqueiro amargoso. }\end{array}$ \\
\hline
\end{tabular}




\section{Resultados}

\section{Lin. Fil.}

Produção científica da base de dados Web of Science com Mauritia flexuosa

A análise da produção científica relacionada com a espécie Mauritia flexuosa Lin. Fil. permitiu observar que foi crescente no período de 1999 a 2017. Foram encontradas 223 publicações produzidas por instituições brasileiras e que a Universidade Estadual de Campinas (UNICAMP), a Universidade Federal do Pará (UFPA), a Universidade de São Paulo (USP), a Universidade de Brasília (UNB), a Universidade Federal do Piauí (UFPI), a Empresa Brasileira de Pesquisa Agropecuária (EMBRAPA), a Universidade Federal da Paraíba (UFPB), a Universidade do Estado de São Paulo (UNESP) e a Universidade Federal do Rio de Janeiro (UFRJ) foram as instituições que publicaram mais artigos sobre a palmeira (Figura 1).
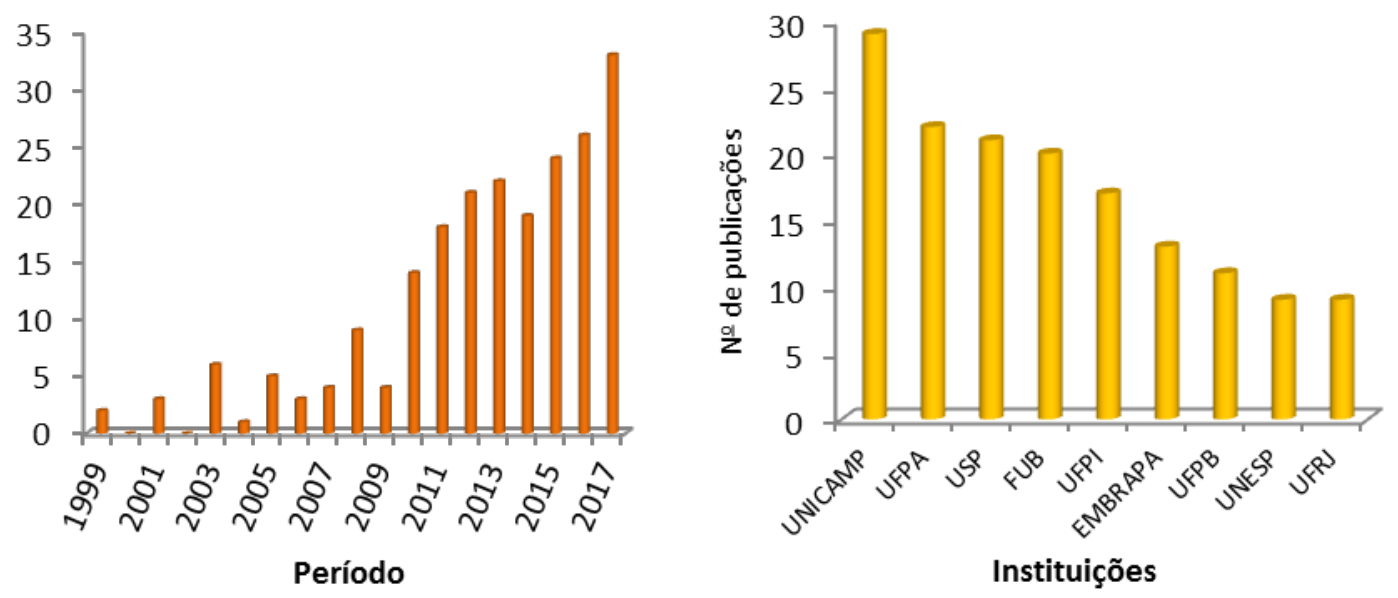

Figura 1. Publicações de artigos sobre buriti no período de 1999 a 2017 (1A) e número de publicações realizadas por instituições brasileiras (1B). Fonte: Web of Science (2019).

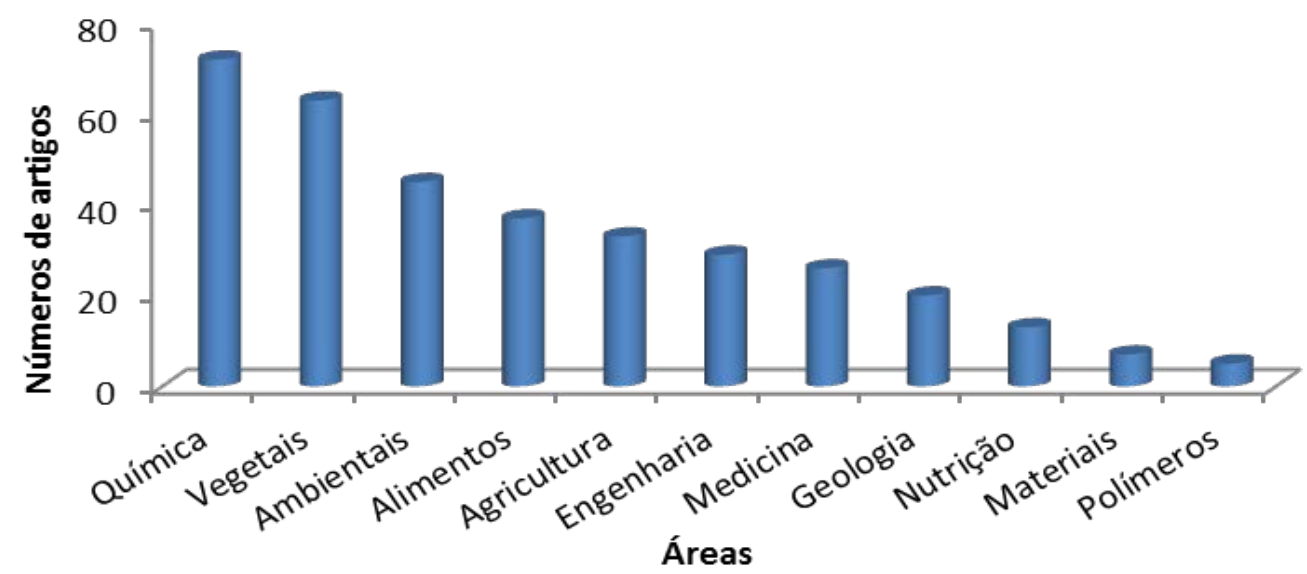

Figura 2. Relação de artigos publicados com buriti em diferentes áreas no período de 1999 a 2017. Fonte: Web of Science (2019). 
As atividades científicas supracitadas em diferentes artigos científicos desenvolvidos permite observar que o Buriti tem grande potencial para desenvolvimento de novos produtos para as indústrias alimentícia, farmacêutica e química. Foi observado publicações nas áreas de Agricultura, Química, Tecnologia da Ciência dos Alimentos, Ciência de Materiais, Engenharia, Medicina, Nutrição Dietética, Ciências Vegetais, Ecologia de Ciências Ambientais, Geologia e Ciência de Polímeros (Figura 2).

Produtos registrados no Instituto Nacional da Propriedade Industrial com Mauritia flexuosa Lin. Fil.

Foram identificados 16 resultados para a espécie Mauritia flexuosa Lin. Fil. no Instituto Nacional da Propriedade Industrial (INPI) no período de 1999 a 2016. Os documentos observados no ano de 2016 podem não corresponder ao número total, visto que a Lei de Propriedade Industrial garante ao depositante o sigilo pelo período de 18 meses, contados a partir da data de depósito (Brasil, 1996). Nos anos de 2000 a 2002, 2006 e 2007, 2010 e 2012 não foram recuperadas patentes. No ano de 2008 foram observados três resultados e nos demais anos foi observada oscilação entre um e dois depósitos (Figura 3A). Considerando o período analisado, de 17 anos, o índice de depósitos realizados ainda é pequeno. É necessário, portanto, esforços para maior uso sustentável da grande biodiversidade de palmeiras que o Brasil possui, por meio do desenvolvimento de novos produtos e processos.

Os resultados das buscas apontam a possibilidade de desenvolvimento tecnológico em campos diversificados, relacionados a produtos industriais (31\%), usos e aplicações em composições cosméticas (25\%), composições químicas para aplicação na indústria (25\%) e produtos alimentícios (19\%). As invenções desenvolvidas estão relacionadas à aplicação dos talos (pecíolo) da palmeira para fabricação de placas, painéis, chapas de compensado e prancha de natação (Figura 3B).
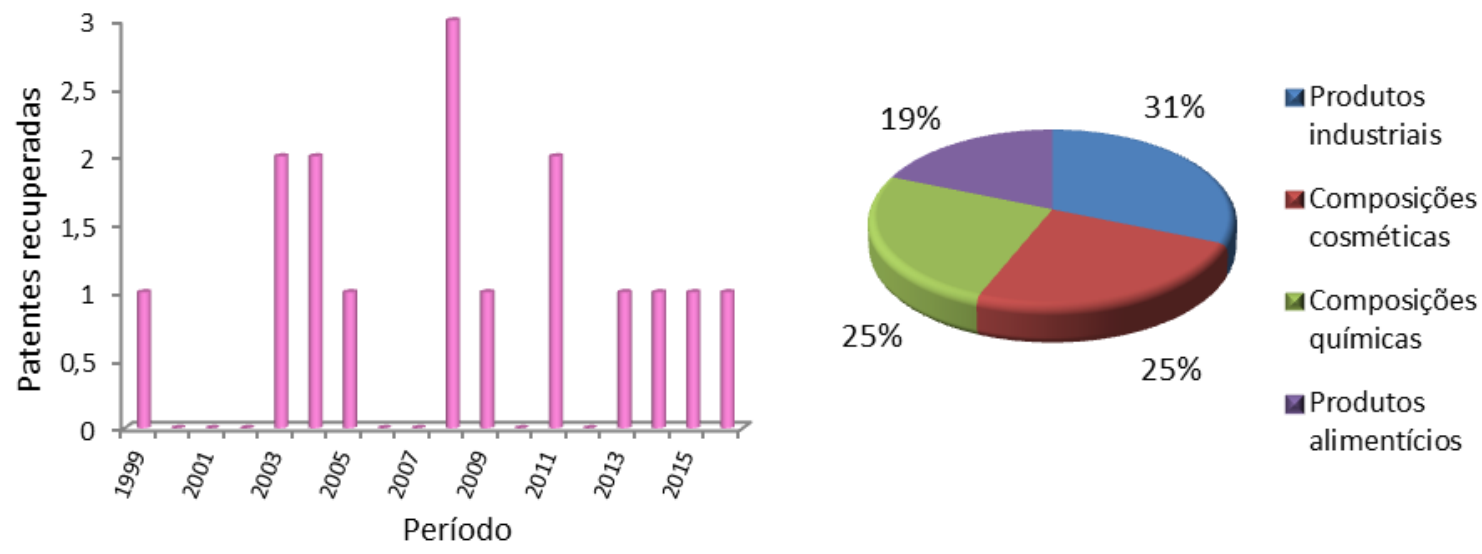

Figura 3. Número de patentes de Mauritia flexuosa Lin. Fil. recuperadas no período de 1999 a 2016 (3A) e áreas por campo tecnológico (3B). Fonte: INPI, 2019.

Produtos registrados no Escritório Europeu de Patentes com Mauritia flexuosa Lin. Fil.

Foram identificados 14 processos depositados para proteção de produtos com utilização da espécie Mauritia flexuosa Lin. Fil. no Escritório Europeu de Patentes 
(ESPACENET) entre os anos de 2000 a 2017. Três processos foram depositados por inventores independentes, três por ICTs (Instituto de Pesquisa da Amazônia) e oito por empresas privadas. Além do Brasil, foram computados depósitos no Escritório Europeu de Patentes, três de residentes (Países Baixos, Alemanha e Reino Unido), um dos Estados Unidos, quatro da China, um das Filipinas e um do Japão.

Produtos registrados no Escritório Latino Americano de Patentes com Mauritia flexuosa Lin. Fil.

Foram observados três processos depositados para proteção de produtos com utilização da espécie Mauritia flexuosa Lin. Fil. junto ao banco do Escritório Latino Americano de Patentes (LATIPAT), nos anos de 2004, 2015 e 2016.

Produção científica da base de dados Web of Science com Acrocomia aculeata (Jacq.) Lodd. ex Mart.

Foram identificados na base de dados Web of Science, 192 publicações relacionadas com a espécie de palmeira Acrocomia aculeata (Jacq.) Lodd. ex Mart. 0 maior número de publicações foi realizado pela Universidade Federal de Viçosa (UFV), seguidas pela Universidade Federal de Minas Gerais (UFMG), Empresa Brasileira de Pesquisa Agropecuária (EMBRAPA), Universidade Estadual de Montes Claros (UNIMONTES), Universidade de Brasília (UnB), Universidade Federal de Mato Grosso do Sul (UFMS), Universidade de São Paulo (USP), Universidade Federal da Grande Dourados (UFGD), Universidade Estadual de Maringá (UEM) e Universidade Federal de Lavras (UFLA) (Figura 4).

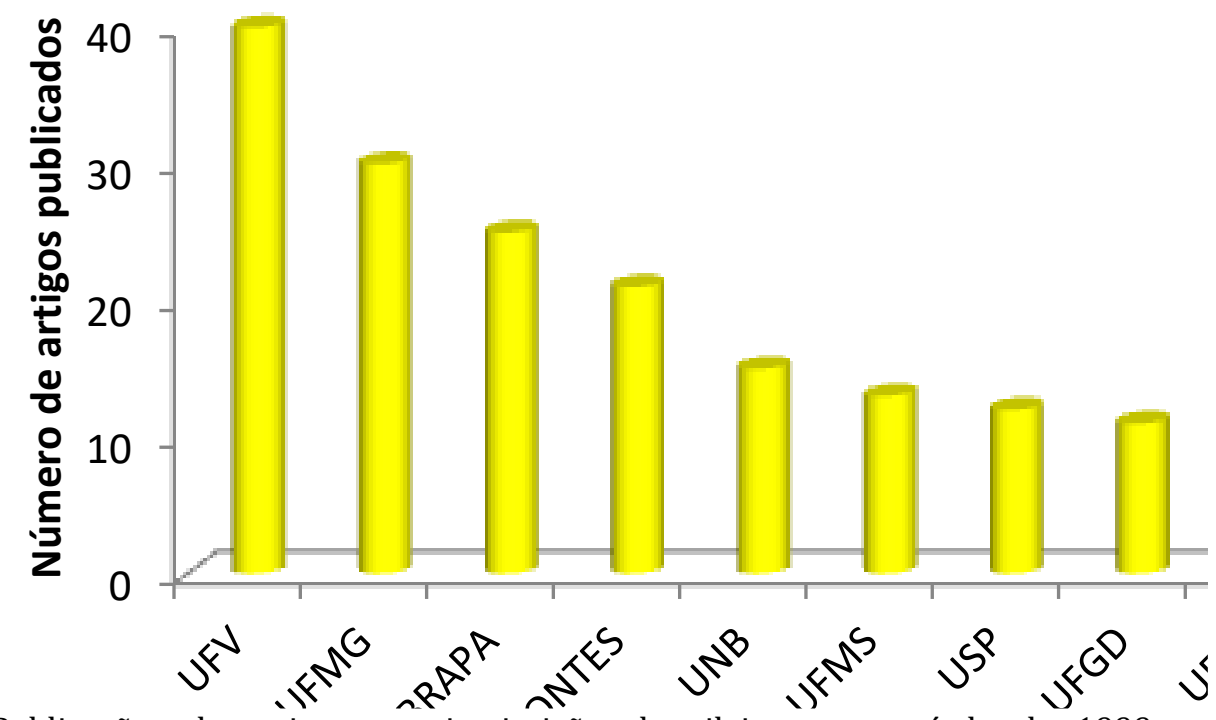

Figura 4. Publicações de artigos por instituições brasileiras no período de 1999 a 2017 recuperados relacionados à macaúba (Acrocomia aculeata (Jacq.) Lodd. ex Mart.). Fonte: Web of Science (2019).

Os resultados obtidos permitiram observar publicações com a espécie Acrocomia aculeata (Jacq.) Lodd. ex Mart. nas áreas de agricultura, botânica, ecologia, ciências ambientais, ciência dos alimentos, microbiologia aplicada à biotecnologia, engenharia, química, combustíveis, bioquímica e biologia molecular e silvicultura (Figura 5). 


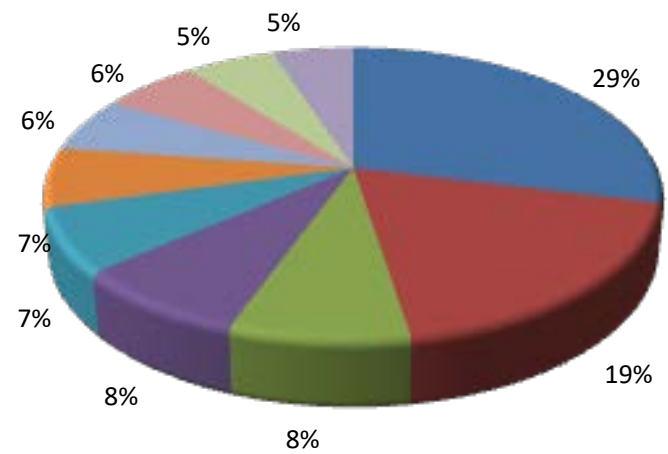

AGRICULTURA

GIÊNCIAS DAS PLANTAS

ECOLOGIA DE CIÊNCIAS AMBIENTAIS

- TECNOLOGIA DA CIÊNCIA ALIMENTAR

a MICROBIOLOGIA APLICADA À BIOTECNOLOGIA

I ENGENHARIA

14 QUÍMICA

a ENERGIA DE COMBUSTÍVEIS

a BIOQUÍMICA BIOLOGIA MOLECULAR

a SILVICULTURA

Figura 5. Publicação de artigos por áreas do conhecimento no período de 1999 a 2017 recuperados no relacionados à macaúba (Acrocomia aculeata (Jacq.) Lodd. ex Mart.). Fonte: Web of Science (2019).

Foram identificados dezenove processos no INPI, entre os anos de 2000 e 2015 com utilização dos termos Acrocomia aculeata e macaúba (Figura 6). Resultados com as palavras macaíba, macaúva, boicaiuva, bocaiúva, coco de catarro, coco baboso, coco de espinho, macajuba, macaibeira, mucaia, mucajá e mucajaba não foram encontrados.

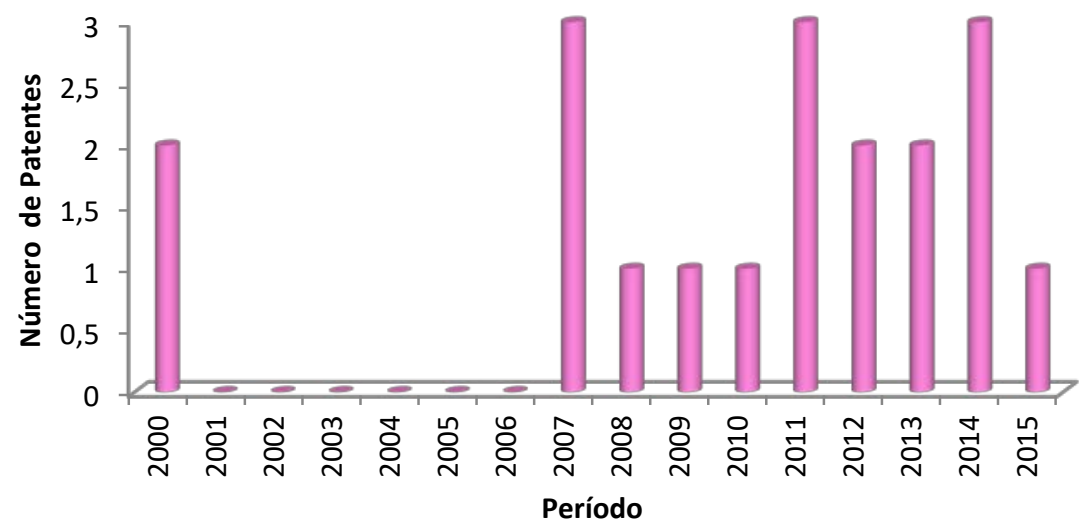

Figura 6. Patentes identificadas na busca tecnológica com Acrocomia aculeata (Jacq.) Lodd. ex Mart. no período de 2000 a 2015. Fonte: INPI (2019).

Produtos registrados no Escritório Europeu de Patentes com Acrocomia aculeata (Jacq.) Lodd. ex Mart.

No Escritório Europeu de Patentes (ESPACENET) foi observado apenas um processo depositado para proteção de produto com utilização do termo Acrocomia aculeata (Jacq.) Lodd. ex Mart. no período de 2000 a 2017. 


\section{(Mart.) Becc. \\ Produção científica da base de dados Web of Science com Syagrus oleracea}

Foram identificados na base de dados Web of Science, 20 publicações científicas realizadas com a espécie Syagrus oleracea (Mart.) Becc. (Figura 7). As principais instituições que realizaram publicações com a espécie foram a Universidade Estadual Paulista (UNESP), a Empresa Brasileira de Pesquisa Agropecuária (EMBRAPA), a Universidade de Brasília (UnB) e a Universidade de São Paulo (USP) (Figura 8).

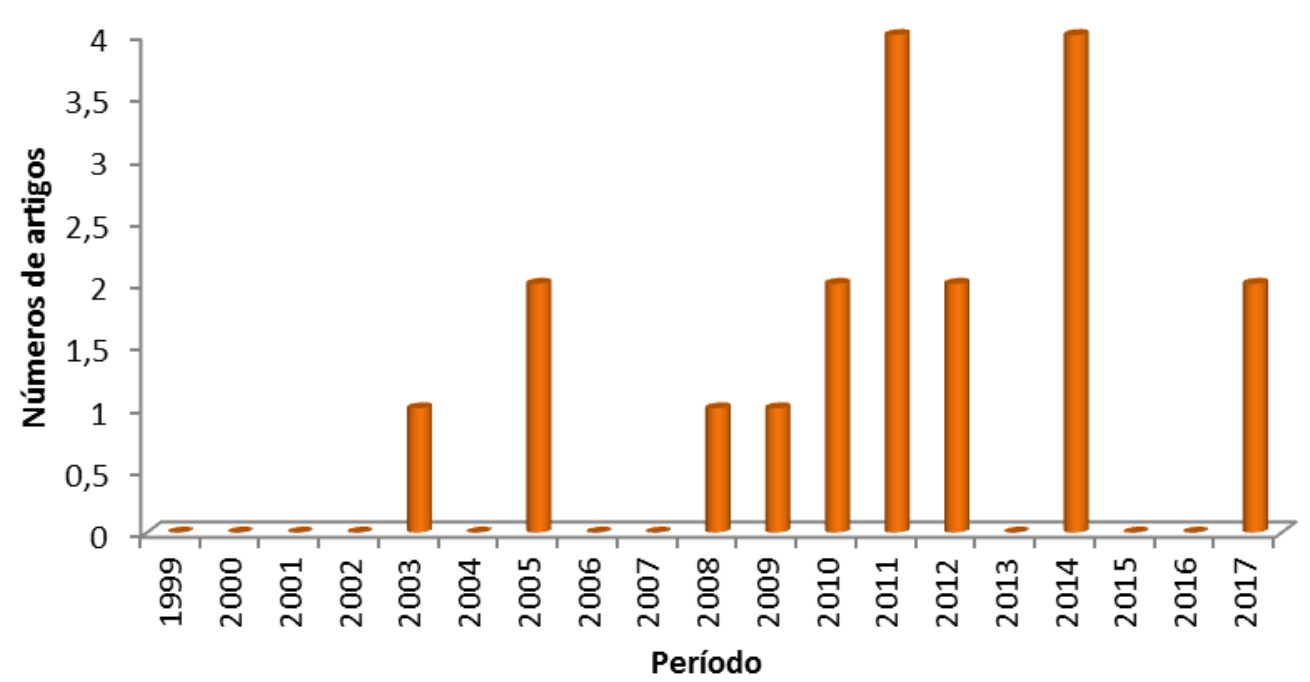

Figura 7. Publicação total de artigos relacionados à palmeira guariroba (Syagrus oleracea (Mart.) Becc.) recuperados no período de 1999 a 2017. Fonte: Web of science, 2019.

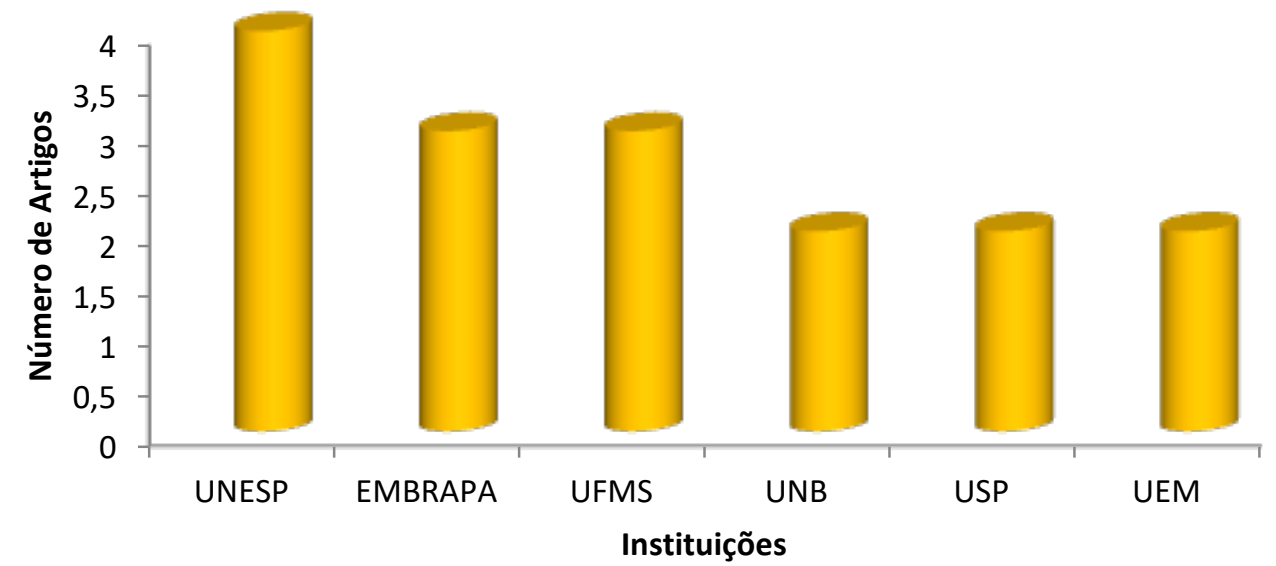

Figura 8. Publicações de artigos por instituições brasileiras no período de 1999 a 2017 recuperados, relacionados à palmeira guariroba Syagrus oleracea (Mart.) Becc. Fonte: Web of science, 2019. 
Os trabalhos foram desenvolvidos principalmente nas áreas da Agricultura, Ciência Alimentar, Química, Ciências das Plantas, Zoologia, Entomologia e Parasitologia (Figura 9).

\section{Produtos registrados no Instituto Nacional da Propriedade Industrial Syagrus oleracea (Mart.) Becc.}

Foram observados dois processos depositados para proteção de produtos com utilização da espécie Syagrus oleracea (Mart.) Becc. junto ao banco de dados do Instituto Nacional de Propriedade Industrial (INPI), com os termos "guariroba" e "Syagrus e oleracea", ambos depositados por inventores independentes.
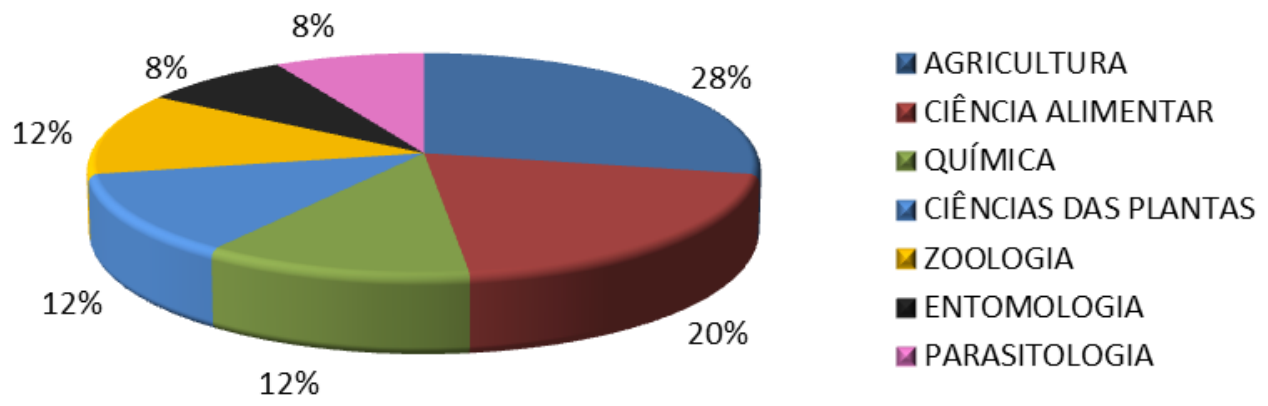

Figura 9. Publicação de artigos por áreas do conhecimento no período de 1999 a 2018 recuperados no relacionados à palmeira guariroba (Syagrus oleracea (Mart.) Becc.). Fonte: Web of Science, 2019.

\section{Discussão}

\section{Lin. Fil.}

Produção científica da base de dados Web of Science com Mauritia flexuosa

Foi observado acréscimo médio anual de 3,7 artigos publicados no período de 1999 a 2009, e média de 22,1 publicações entre o período de 2010 a 2017 com o buriti (Figura 1). 0 crescimento do número de publicações evidencia o interesse no buriti como matéria-prima para estudos científicos e desenvolvimento de produtos para diferentes aplicações. Foram observados trabalhos científicos desenvolvidos com o buriti para diferentes finalidades. Ao ser analisado trabalhos para determinação do perfil fitoquímico e as atividades nutricionais e farmacológicas da espécie Mauritia flexuosa Lin. Fil., os principais compostos bioativos encontrados foram carotenóides, tocoferóis, ácido ascórbico, compostos fenólicos, fibras, fitoesteróis e ácidos graxos mono e poli-insaturados. Os compostos supracitados estão relacionados principalmente com atividades antioxidante, hipolipemiante, fotoprotetor, antiagregante, antitrombótico, antiinflamatório, hipoglicemiante, antimicrobiano e antitumoral (Freire et al., 2016). Foi realizado também em trabalho desenvolvido com o buriti, o desenvolvimento de extração e caracterização da proteína polifenol oxidase, avaliação da estabilidade térmica e a possibilidade de utilização da espécie de palmeira na construção de biossensores (Carvalho, 2017).

Ao ser analisado artigos que tinham como finalidade a caracterização completa do óleo do buriti, foram identificados como principais compostos o $\beta$-caroteno, o $\alpha$-tocoferol, $\beta$-tocoferol e os ácidos graxos monoinsaturados. Foi observado que o óleo de buriti pode 
ser alternativo para o enriquecimento de alimentos com compostos bioativos (Freitas et al., 2017). Foram avaliadas também nanoemulsões do óleo de buriti para incorporação em bebidas isotônicas e para substituição da coloração artificial por corantes naturais e obtidos resultados positivos (Bovi et al., 2017). Foi demonstrado também que os frutos do buritizeiro apresentam alta atividade antioxidante e quantidade razoável de compostos fenólicos, e carotenóides (Milanez et al., 2018).

Ao analisar os artigos relacionados à área de química é observado o interesse de identificação de produtos ou processos que auxiliem na recuperação ou preservação do meio ambiente. Existem estudos com o fruto da palmeira buriti na busca de materiais biodegradáveis utilizado na remoção de metais de efluentes industriais (Pinto, 2013) e com a casca dos frutos para ser utilização como agente ativador e removedor do azul de metileno de soluções aquosas (Pezoti et al., 2014). Foi observado trabalho com a Mauritia flexuosa Lin. Fil. para compreensão em relação ao desenvolvimento dos frutos em função da localização geográfica e do quantitativo de polinizadores com objetivo de estabelecer estratégias para aumentar a produtividade. Foi avaliado de forma experimental, o sistema reprodutivo e os visitantes florais presentes no processo de polinização de flores de Buritizeiro em ambiente de restinga costeira no nordeste do Brasil (Mendes et al., 2017). Foi proposto também trabalho para determinação do valor ambiental da palmeira Buriti de acordo com a disposição máxima a ser pago para conservação da espécie, no sentido de preservação da floresta à medida que se confronta os valores envolvidos nas atividades extrativistas com os valores pagos para conservação do meio ambiente (Vieira, 2016).

Estudos que relacionam a Mauritia flexuosa Lin. Fil. e o ambiente visaram analisar, além das condições ambientais, a importância socioeconômica, os benefícios sociais da utilização dos frutos e os possíveis potenciais de utilização da espécie. A palmeira é amplamente distribuída na América do Sul e está associada a ambientes inundados, a ecossistemas pantanosos e a veredas do bioma Cerrado. Os frutos oleaginosos são importantes para a manutenção da fauna regional e utilizados como fonte de renda para populações tradicionais (Porto, 2017). Foram observadas aplicações da espécie Mauritia flexuosa Lin. Fil. na indústria alimentícia, como a elaboração de geleia de Buriti que possui aspecto físicoquímico, fibras, valor energético e vitaminas lipossolúveis, apresentaram potencial antioxidante, além de consideráveis teores de vitaminas A e $\mathrm{D}$, podendo também ser considerada fonte de fibras.

\section{Produtos registrados no Instituto Nacional da Propriedade Industrial}

As Instituições Científicas e Tecnológicas (ICTs) identificadas como titulares no INPI, detêm $50 \%$ dos depósitos, com destaque para a Universidade Estadual de Campinas (UNICAMP), com três depósitos. A Fundação Assis Gurgaz (FAG), a Fundação Universidade de Brasília (UnB), a Universidade Estadual do Maranhão (UEMA), o Instituto Nacional de Pesquisa da Amazônia (INPA) e o Polo de Proteção da Biodiversidade e Uso Sustentável dos Recursos Naturais (POLOPROBIO), cada um com um depósito de patente. Juntas essas instituições possuem um total de oito processos de depósito de patentes, seguido de seis para inventores independentes e dois para empresas privadas, todos residentes no Brasil.

Os produtos foram desenvolvidos em campos diversificados, relacionados a produtos industriais, usos e aplicações em composições cosméticas, composições químicas para aplicação na indústria e produtos alimentícios. Os pedidos referentes a produtos industriais apresentaram o maior percentual dos documentos de patentes depositados, correspondente a 31\%. As invenções desenvolvidas estão relacionadas à aplicação dos talos (pecíolo) da palmeira para fabricação de placas, painéis, chapas de compensado e prancha de natação (Figura 3B).

As patentes que descrevem produtos e/ou processos a partir do uso do óleo dos frutos e das sementes da palmeira (25\%) trazem informações que possibilitam a análise das propriedades e aplicações. A PI 0403407-4, concedida em 2014, desenvolvida por

Rev. Bras. Gest. Amb. Sustent., 2020, vol. 7, n. 17, p. 1335-1352. 
pesquisadores da Universidade de Brasília protege a preparação de compósitos com propriedades fotoprotetoras. A BR 102013033412-0, depositado em 2013 pela UNICAMP, refere-se ao desenvolvimento de nanopartículas associado ao óleo da polpa do Buriti com eficácia no tratamento de alopecia (calvície). 0 processo PI 0303404-6, depositado em 2003 pela empresa Chemyunion, requereu a proteção de uso do óleo da polpa do Buriti para preparação de formulações cosméticas, capilares e farmacêuticas e como potencializador de proteção solar. 0 referido processo tornou-se de domínio público, após 10 anos de tramitação, por não cumprir exigências da lei brasileira quanto à declaração de acesso ao patrimônio genético, junto ao INPI. A Lei no 13.123/2015 (Lei de Acesso ao Patrimônio Genético e ao Conhecimento Tradicional Associado) dispõe que produtos desenvolvidos com uso de parte ou todo de seres da fauna, flora e ao conhecimento tradicional, no depósito para proteção de patentes junto ao INPI, devem ser anexados autorização de acesso ao patrimônio genético, emitida pelo Conselho de Gestão do Patrimônio Genético (CGEN), sob pena de arquivamento.

No que concerne à análise da situação legal, um processo de patente foi concedido, um indeferido, sete publicados e sete arquivados. Entre os sete processos arquivados, cinco correspondem a depositantes independentes, um a ICT e um a empresa privada. Os inventores independentes apareceram na segunda colocação em depósitos, no entanto, há maior ocorrência de abandono das patentes por estes. As análises das publicações processuais de cada patente revelaram que os fatores de abandono estão relacionados à falta de pagamento da anuidade, descumprimento de exigências e falta de pedido de exame no prazo de 36 meses contados da data do depósito, conforme exigência da lei.

Os processos de patentes depositados por pessoas físicas, conforme consta na primeira página ou folha de rosto do documento, em sua maioria, são protocolados pelos próprios inventores, sem a assessoria de profissionais da área de propriedade intelectual, diferente das ICTs, que são assessoradas pelos Núcleos de Inovação Tecnológica, responsáveis pela gestão da política de inovação, e das empresas que constituem procuradores para acompanhar os processos, fator relevante em relação a aferição dos abandonos e processos em andamento.

\section{Produtos registrados no Escritório Europeu de Patentes}

Foram observados processos foram depositados por inventor independente, por ICT (Instituto de Pesquisa da Amazônia) e por empresas privadas. Além do Brasil, foram computados depósitos no Escritório Europeu de Patentes, três de residentes (Países Baixos, Alemanha e Reino Unido), um dos Estados Unidos, quatro da China, um das Filipinas e um do Japão. Diferentemente dos resultados observados no INPI, em que ocorreu a presença de depósito de produtos de campos tecnológicos distintos, no ESPACENET todos os processos observados foram relacionados a produtos de higiene pessoal e cosméticos. Outro aspecto relevante diz respeito à titularidade dos depositantes internacionais que são majoritariamente empresas privadas.

\section{Produtos registrados no Escritório Latino Americano de Patentes}

Os processos foram depositados por uma empresa privada do Brasil, uma empresa privada dos Estados Unidos e por uma universidade do Peru. No Brasil a empresa Natura Cosméticos S.A. foi responsável por um depósito (PI 0201235-9) referente a composição cosmética. 0 processo da Natura Cosméticos S.A. foi depositado com prioridade brasileira e em seguida ocorreu o depósito internacional subsequente no Peru.

A Natura Cosméticos S. A. historicamente dedica-se à produção de cosméticos de formulação natural, para cuidados com a pele, cabelos e corpo e investe em pesquisas de novos produtos e tecnologias com utilização da biodiversidade brasileira (Natura, 2011). Apresenta um amplo portfólio de produtos, mas, para o Buriti foi recuperada uma patente. 
Total de produtos registrados com uso da espécie Mauritia flexuosa Lin. Fil.

De todos os processos registrados com a espécie Mauritia flexuosa Lin. Fil. que atenderam à finalidade da pesquisa, dez foram desenvolvidos por ICTs, quatro por inventores independentes, onze por empresas privadas e ocorreu a incidência de oito arquivamentos, cuja matéria objeto de proteção encontra-se em domínio público. Um dado relevante é que das 11 empresas depositantes com processos válidos, nove são internacionais e duas apenas são brasileiras.

Uma das empresas privadas internacionais responsáveis pelos depósitos de produtos com utilização da espécie Mauritia flexuosa Lin. Fil. é a alemã Coty Inc., empresa global que fabrica cosméticos e inclui no portfólio marcas de renome mundial como Marc Jacobs, Calvin Klein, Chloé, Gucci, Hugo Boss, Tiffany \& Co, Balenciaga, Bottega Veneta, Alexander McQueen, Davidoff, Burberry e Miu Miu. As outras empresas, conforme informações constantes nos sítios eletrônicos, são responsáveis por depósitos com utilização da espécie supracitada, produzem, vendem e exportam produtos de higiene pessoal e cosméticos à base de plantas. Foram identificadas as empresas Medical Brands e Aveda Corp dos Estados Unidos, Beijing Huaao Guangcheng Cosmetics Co LTD, Beijing Huaao Optical Path Cosmetic Co LTD e Anhui Huituoqing Biotechnology Co LTD da China, Dr. Scheller Cosmetics AG da Alemanha, Ichimaru Pharcos do Japão e a australiana Jurlique.

\section{Produção científica da base de dados Web of Science com Acrocomia aculeata (Jacq.) Lodd. ex Mart.}

Os resultados obtidos permitem observar que a área da pesquisa que ocorreu maior número de publicações é a Agricultura (29\%), enquanto áreas na fronteira da tecnologia, a exemplo da Microbiologia aplicada à Biotecnologia (7\%), que visa aplicar os conhecimentos científicos para o uso de microrganismos com potencial de gerar produtos e/ou processos de interesse comercial, ainda precisam avançar (Figura 7).

Produtos registrados no Instituto Nacional da Propriedade Industrial Acrocomia aculeata (Jacq.) Lodd. ex Mart.

Para a espécie de palmeira Acrocomia aculeta, a maior quantidade de depositantes observados foram ICTs. Nove produtos foram depositados por universidades brasileiras (Universidade Federal de Minas Gerais, Universidade Federal de Viçosa e Universidade Estadual de Montes Claros), cinco produtos depositados por inventores independentes e cinco produtos depositados por empresas privadas.

Ao considerar o campo tecnológico das patentes desenvolvidas para a espécie Mauritia flexuosa Lin. Fil., os documentos observados descrevem produtos relacionados à alimentação humana e animal, processamento de materiais, produção de biocombustível, produção de carvão, desenvolvimento de produtos industriais, de produtos cosméticos, de maquinário para descascar, despolpar e processar frutos, ferramenta de perfuração da semente para permitir a germinação e também processo para otimização da germinação de sementes. Foi observado o desenvolvimento de várias aplicações tecnológicas com utilização do fruto, do óleo, da polpa e da semente e do endocarpo da espécie Mauritia flexuosa Lin. Fil.

A empresa Petróleo Brasileiro S.A. (PETROBRAS), que desenvolve trabalhos de pesquisa e tecnologias inovadoras em parceria com diferentes ICTs, foi responsável pelo maior número de depósitos no INPI. Os produtos desenvolvidos pela PETROBRAS não ficam restritos à produção de biocombustível.

Ao serem analisadas as situações dos processos depositados no INPI foi possível constatar que cinco foram arquivados, dez publicados e um concedido. Entre os cinco processos arquivados, quatro foram depositados por pessoas físicas e um por empresa

Rev. Bras. Gest. Amb. Sustent., 2020, vol. 7, n. 17, p. 1335-1352. 
privada. A incidência de abandonos de patentes ocorre por falta de pagamentos das anuidades ou por faltas de pedidos de exames.

O processo PI 0003920-9, referente a "Formulação para tratamento de distúrbios capilares", apresentou uma peculiaridade em relação a todos os processos recuperados, concedida para inventora independente em 2005, foi o único que apresentou anotação de transferência de tecnologia para a empresa "Florevero Indústria e Comércio de Cosméticos Ltda. ME", com sede no município de São Paulo, estado de São Paulo.

Para as ICTs, a transferência de tecnologia enfrenta barreiras, porque significativa parcela dos pesquisadores desenvolve pesquisas e publicam resultados por não terem viés tecnológico. Mesmo quando a patente é depositada, os pesquisadores dependem de Núcleos de Inovação Tecnológica presentes nas instituições para construção de estratégias para oferta das tecnologias e busca de potenciais interessados, quando o caminho poderia ser percorrido a partir da verificação do estado da arte e as demandas do mercado para desenvolvimento de produtos e processos em parceria.

\section{Produtos registrados no Escritório Europeu de Patentes}

0 processo identificado trabalha com concentrações de matérias primas da espécie Mauritia flexuosa Lin. Fil. e Acrocomia crispa (Kunth) C. F. Baker ex Becc. que é endêmica de Cuba. O depósito de patente (CU20120000097) foi realizado pelo Centro Nacional de Investigaciones Científicas (CNIC), com sede na República Cubana. A patente de invenção refere-se a um método de obtenção de princípio ativo a partir dos frutos verdes ou maduros das palmeiras, para ser usado como suplemento nutricional, em formulações cosméticas, terapêuticas ou em composições farmacêuticas, para prevenir e ou tratar processos inflamatórios e estresse oxidativo, que acompanham o processo de envelhecimento e muitas patologias, como a hiperplasia benigna de próstata e osteoartrite. A patente foi depositada inicialmente em Cuba e posteriormente no México, Austrália, Colômbia, Canadá, China, Dinamarca, Escritório Europeu, Índia, Japão, República da Coréia, Noruega, Filipinas e Estados Unidos.

\section{Produção científica da base de dados Web of Science com Syagrus oleracea} (Mart.) Becc.

Foram identificados na base de dados Web of Science, 20 publicações científicas realizadas com a espécie Syagrus oleracea (Mart.) Becc.(Figura 8). As principais instituições que realizaram publicações com a espécie foram a Universidade Estadual Paulista (UNESP), a Empresa Brasileira de Pesquisa Agropecuária (EMBRAPA), a Universidade de Brasília (UnB) e a Universidade de São Paulo (USP) (Figura 9). As linhas de pesquisa que desenvolvem trabalhos científicos com a espécie Syagrus oleracea (Mart.) Becc. provavelmente devem ter iniciado recentemente. Os trabalhos são desenvolvidos principalmente em atividades de descrição, identificação, trabalhos com germinação de sementes e de caracterização do óleo vegetal para uso industrial, conforme demonstram os resultados recuperados na plataforma Web of Science. Os resultados obtidos permitem observar que esta espécie pode ser objeto futuro de estudos e publicações científicas em diversas áreas, visto que foram recuperados pequeno número de produções.

\section{Produtos registrados no Instituto Nacional da Propriedade Industrial com Syagrus oleracea (Mart.) Becc.}

Foram observados dois produtos desenvolvidos e depositados com utilização da espécie Syagrus oleracea (Mart.) Becc. junto ao banco de dados do Instituto Nacional de Propriedade Industrial (INPI). 0 primeiro refere-se a creme hidratante, com ação antienvelhecimento a base de óleo de guariroba, seguindo forte tendência da utilização de produtos baseados em matérias primas decorrentes de fontes naturais. 0 segundo refere a papel ecológico feito a partir de material orgânico ou resíduos não utilizáveis da casca da 
guariroba. No entanto, os dois processos foram arquivados pelo INPI e encontram-se em domínio público.

\section{Conclusões}

O Brasil é o maior gerador de conhecimento científico relacionado às espécies de palmeiras supracitadas, em áreas relacionadas especialmente à agricultura, botânica e ciência dos alimentos, com destaque para a participação da Universidade Estadual de Campinas (UNICAMP), Universidade Federal de Viçosa (UFV) e Universidade do Estado de São Paulo (UNESP).

Tanto as atividades científicas quanto os desenvolvimentos de produtos com utilização das palmeiras Mauritia flexuosa Lin. Fil., Acrocomia aculeata (Jacq.) Lodd. ex Mart. e Syagrus oleracea (Mart.) Becc. como matéria-prima, são desenvolvidos em diversas áreas do conhecimento.

Nas ICTs tem prevalecido a publicação de artigos científicos e nas indústrias, mesmo que de forma incipiente, observam-se solicitações de patentes, com o objetivo de recuperar financeiramente os investimentos em pesquisas e desenvolvimentos tecnológicos. Para o caso das palmeiras do cerrado brasileiro, especialmente para o buriti, são as empresas europeias (Países Baixos, Alemanha e Reino Unido), norte americanas, chinesas, filipinas, japonesas, alemãs e australianas, que investem em pesquisa e desenvolvimento principalmente nas áreas de higiene pessoal e cosmetologia.

Apesar do grande potencial para desenvolvimento de produtos, as espécies de palmeiras estudadas não têm sido muito utilizadas para geração de inovações pelas empresas brasileiras. Mais investimentos em ciência, tecnologia e inovação e maior estabelecimento de parcerias das ICTs com o setor empresarial são ações necessárias para ampliar a geração de produtos de maior valor agregado. A transferência das novas tecnologias geradas são ações que contribuirão com o desenvolvimento do país, com a geração de empregos, de renda e de impostos para melhor atendimento das necessidades da sociedade brasileira.

\section{Agradecimentos}

Ao Programa de Pós-Graduação em Biotecnologia da Universidade Estadual de Montes Claros, à Fundação de Amparo à Pesquisa do Estado de Minas Gerais (FAPEMIG) pela Bolsa de Gestão em Ciência, Tecnologia e Inovação concedida a Roseli Aparecida Cardoso Vieira e Medeiros e ao Núcleo de Propriedade Intelectual e Inovação Tecnológica da Universidade Estadual de Montes Claros.

\section{Conflito de interesses}

Os autores declaram não haver conflitos de interesse.

\section{Referências}

Abreu, N. A. Cultura da guariroba: uma produção constante e rentável. 3. ed. Goiânia: Aeago, 1997.

Arellano, D. B.; Silva, C. R.; Velazquez, M. C; Polezel, M. A.; Nogueira, C. Uso do óleo da polpa dos frutos de palmeiras do gênero Mauritia, como potencializador da protecção solar e como fonte de carotenoides (pró-vitamina A) e vitamina E (tocoferois) antioxidantes naturais em produtos cosméticos, capilares e farmacêuticos. PI patente 0303404-6. 23 dez. 2003. 
Barcelos, E.; Rodrigues, F. M.; Morales, E. A.V. Dendeicultura: alternativa para o desenvolvimento sustentável no Amazonas. Manaus: EMBRAPA Amazônia Ocidental, 1999.

Bhering, L. Macaúba: matéria-prima nativa com potencial para a produção de biodiesel. 2018. Disponível em: <http://www.diadecampo.com.br/zpublisher/materias/Materia.asp ?id=21272\&secao=Artigos Especiais > . Acesso em: 20 out. 2019.

Bovi, G. G.; Petrus, R. R.; Pinho, S. C. Feasibility of incorporating buriti (Mauritia flexuosa L.) oil nanoemulsions in isotonic sports drink. International Journal of Food Science \& Technology, v. 52, p. 2201-2209, 2017. https://doi.org/10.1111/ijfs.13499

Brasil. Lei no 9.279, de 14 de maio de 1996. Regula direitos e obrigações relativos à propriedade industrial. Disponível em: <http://www.planalto.gov.br/ccivil_03/leis/ 19279.htm>. Acesso em: 20 out. 2019.

Brasil. Lei no 13.123, de 20 de maio de 2015. Regulamenta o inciso II do $§ 1^{\text {o }}$ e o $\S 4^{\circ}$ do art. 225 da Constituição Federal, o Artigo 1, a alínea j do Artigo 8, a alínea c do Artigo 10, o Artigo 15 e os $\S \S 3^{\circ}$ e $4^{\circ}$ do Artigo 16 da Convenção sobre Diversidade Biológica, promulgada pelo Decreto no 2.519, de 16 de março de 1998; dispõe sobre o acesso ao patrimônio genético, sobre a proteção e o acesso ao conhecimento tradicional associado e sobre a repartição de benefícios para conservação e uso sustentável da biodiversidade; revoga a Medida Provisória no 2.186-16, de 23 de agosto de 2001; e dá outras providências. Disponível em: <http://www.planalto.gov.br/ccivil_03/_ato2015-2018/ 2015/lei/l13123.htm>. Acesso em: 20 out. 2019.

Brasil. Lei no 13.243, 11 de janeiro de 2016. Dispõe sobre estímulos ao desenvolvimento científico, à pesquisa, à capacitação científica e tecnológica e à inovação e altera a Lei $\mathrm{n}^{0}$ 10.973, de 2 de dezembro de 2004, a Lei no 6.815, de 19 de agosto de 1980, a Lei no 8.666, de 21 de junho de 1993, a Lei no 12.462, de 4 de agosto de 2011, a Lei no 8.745, de 9 de dezembro de 1993, a Lei no 8.958, de 20 de dezembro de 1994, a Lei no 8.010, de 29 de março de 1990, a Lei no 8.032, de 12 de abril de 1990, e a Lei no 12.772, de 28 de dezembro de 2012, nos termos da Emenda Constitucional no 85, de 26 de fevereiro de 2015. Disponível em: <http://www.planalto.gov.br/ccivil_03/_ato2015-2018/2016/ lei/l13243.htm>. Acesso em: 20 out. 2019.

Caballero, N. E.; Caversan, J.; Gaspari, P. D. M. Nonopartícula lipídica carreadora e seu uso. BR patente 102013033412-0. 15 abr. 2014.

Carvalho, J. C.; Souza, A. L; Machado, C. C. Ecologia, manejo, silvicultura e tecnologia da macaúba. Convenção de Cooperação Tecnológica SETES/FAPEMIG. Polo de Excelência em Floresta. UFV, Viçosa, 2011.

Carvalho, J. O.; Orlanda, F. F. Heat stability and effect of $\mathrm{pH}$ on enzyme activity of polyphenol oxidase in buriti (Mauritia flexuosa Linnaeus f.) fruit extract. Food Chemistry, v. 233, p. 159-163, 2017. https://doi.org/10.1016/j.foodchem.2017.04.101

CNIC CT NAC Investigaciones. Compounds from the fruits of Acrocomia crispa and Acrocomia aculeata for use against oxidative stress and inflammation. CU patente 20120097. 19 jun. 2012.

Dias, J. E. Cadeia produtiva do óleo de Gueroba (Syagrus oleracea BECC.): geração de renda para agricultores familiares e promoção da agrobiodiversidade. Revista Brasileira de Agroecologia, v.9, p.122-133, 2014.

ESPACENET - European Patent Office (EPO). Disponível em: <https://worldwide.espacenet.com>. Acesso em: 27 abr. 2019. 
Freire, J. A. P.; Barros, K. B. N. T.; Lima, L. K. F.; Martins, J. M.; Araújo, Y. C.; Oliveira, G. L. S.; Aquino, J. S.; Ferreira, P. M. P. Phytochemistry profile, nutritional properties and pharmacological activities of Mauritia flexuosa. Journal of Food Science, v. 81, p. 2611-2622, 2016. https://doi.org/10.1111/1750-3841.13529

Freitas, M. L. F.; Chiste, R. C.; Polachini, T. C.; Sardella, L. A. C. Z.; Aranha, C. P. M.; Ribeiro, A. P. B.; Nicoletti, V. R. Quality characteristics and thermal behavior of buriti (Mauritia flexuosa L.) oil. Grasas y Aceites, v. 68, p. 1-9, 2017. https://doi.org/10.3989/ gya.0557171

INPI - Instituto Nacional de Propriedade Industrial. Disponível em: <http://www.inpi.gov.br/menu-servicos/patente/guia-completo-de-patente>. Acesso em: 10 maio 2018.

LATIPAT-Patentes da América Latina e Espanha. Disponível em: <https://lp.espacenet.com>. Acesso em: 28 jul. 2018.

Leonardi, G. R. Cosmetologia aplicada. São Paulo: Medfarma, 2004.

Mendes, F. N.; Valente, R. D.; Rego, M. M. C.; Esposito, M. C. The floral biology and reproductive system of Mauritia flexuosa (Arecaceae) in a restinga environment in Northeastern Brazil. Brittonia, v. 69, p. 11-25, 2017. https://doi.org/10.1007/s12228016-9444-2

Milanez, J. T.; Neves, L. C.; Colombo, C.; Shahab, M.; Roberto, S. R. Bioactive compounds and antioxidant activity of buriti fruits, during the postharvest, harvested at different ripening stages. Scientia Horticulturae, v. 227, p.10-21, 2018. https://doi.org/10.1016/ j.scienta.2017.08.045

Motta, P. E. F.; Curi, N.; Oliveira-Filho, A. T.; Gomes, J. B. V. Ocorrência da macaúba em Minas Gerais: relação com atributos climáticos, pedológicos e vegetacionais. Pesquisa Agropecuária Brasileira, v. 37, p. 1023-1031, 2002. https://doi.org/10.1590/S0100204X2002000700017

Murta, M. M.; Drummond, A. L.; Durães, J. A.; M.J.A., Moreira, S. G. C.; Pimentel, T. A. P. F. Processo para a preparação de compósitos fotoprotetores e fotoluminescentes a partir da dopagem de poliestireno e poli (metacrilato de metila) com óleo de buriti (Mauritia flexuosa L.). PI patente 0403407-4. 05 ago. 2014.

Natura. Parcerias para ciência e tecnologia. Disponível em: <http://www.relatoweb.com.br/natura/13/sites/default/files/ra_2011_completo_gri_por t_0.pdf >. Acesso em: 10 jun. 2018.

Nunes, A. A. Óleo da polpa da macaúba (Acrocomia aculeata (Jacq.) Lodd.) com alta qualidade: processo de refino e termoestabilidade. MS: Universidade Católica Dom Bosco, 2013. (Dissertação de mestrado).

Oliveira, H. C. B. Diretrizes e recomendações técnicas para adoção de boas práticas de manejo para o extrativismo do fruto da macaúba (bocaiúva). Brasília: MDA, 2014. (Cartilha do Programa Nacional de Produção e Uso de Biodiesel - PNPB PNUD/SAF - BRA 11/009/1, do Ministério do Desenvolvimento Agrário - MDA).

Pagano, E. L. P. Composição cosmética multifásica. PI patente 0201235-9. 02 maio 2017.

Pezoti, O.; Cazetta, A. L.; Souza, I. P. A. F.; Bedin, K. C.; Martins, A. C.; Silva, T. L.; Almeida, V. C. Adsorption studies of methylene blue onto $\mathrm{ZnCl}_{2}$-activated carbon produced from buriti shells (Mauritia flexuosa L.). Journal of Industrial and Engineering Chemistry, v. 20, p. 4401-4407, 2014. https://doi.org/10.1016/j.jiec.2014.02.007 
Pinto, M. V. D.; Silva, D. L.; Saraiva, A. C. F. Production and characterization of the activated carbon from buriti stone (Mauritia flexuosa L. f.) to evaluate the adsorption's process of copper (II). Acta Amazonica, v. 43, p.73-80, 2013. https://doi.org/10.1590/S004459672013000100009

Pires, T. P.; Souza, E. S.; Kuki, K. N.; Motoike, S. Y. Ecophysiological traits of the macaw palm: A contribution towards the domestication of a novel oil crop. Industrial Crops and Products, v. 44, p. 200-210, 2013. https://doi.org/10.1016/j.indcrop.2012.09.029

Porto, K. C. N.; Nunes, Y. R. F.; Ribeiro, L. M. The dynamics of recalcitrant seed banks of Mauritia flexuosa (Arecaceae) reveal adaptations to marsh microenvironments. Plant Ecology, v. 219, p. 199-207, 2017. https://doi.org/10.1007/s11258-017-0788-9

Roscoe, R. R. A.; Maranho, E. Análise de viabilidade técnica de oleaginosas para produção de biodiesel em Mato Grosso do Sul. Revista Política Agrícola, v. 16, p. 48-59, 2007.

Rosso, V. V.; Mercadante, A. Z. Identification and quantification of carotenoids, by HPLCPDA-MS/MS, from Amazonian fruits. Journal of Agricultural and Food Chemistry, v. 55, p. 5062-5072, 2007. https://doi.org/10.1021/jf0705421

Silva, D. B.; Martins, R. C.; Agostini-Costa, T. S. Buriti. Jaboticabal: FUNEP, 2010. (Série frutas nativas).

Siqueira, P. B. Caracterização bioquímica e compostos bioativos de macaúba (Acrocomia aculeata (Jacq.) Lodd ex Mart.). Campinas: Universidade Estadual de Campinas, 2012. (Tese de doutorado).

Speziali, M. G.; Sinisterra, R. D. Buscas de informações tecnológicas com base em dados de patentes: estudo de caso dos líquidos iônicos no Brasil. Química Nova, v. 38, p. 1132-1138, 2015. https://doi.org/10.5935/0100-4042.20150126

Vieira, N. V. Formulação para o tratamento de distúrbios capilares. PI patente 0003920-9, 16 ago. 2005.

Vieira, I. R.; Oliveira, J. S.; Santos, K. P. P.; Silva, G. O.; Vieira, F. J.; Barros, R. F. M. A contingent valuation study of buriti (Mauritia flexuosa L.f.) in the main region of production in Brazil: Is environmental conservation a collective responsibility? Acta Botanica Brasilica, v. 30, p. 532-539, 2016. https://doi.org/10.1590/0102$33062016 a b b 0115$

Web of Science. Base de dados. Thomson Reuters. Disponível em: <http://images.webofknowledge.com/images/help/pt_BR/WOK/hp_database.html\#dsy3 66-TRS_wos>. Acesso em: 20 ago. 2018.

Zanatta, C. F.; Ugartondo, V.; Mitjans, M.; Rocha Filho, P. A.; Vinardell, M. P. Low cytotoxicity of creams and lotions formulated with buriti oil (Mauritia flexuosa L.) assessed by the neutral red release test. Food and Chemical Toxicology, v. 46, p. 2776-2781, 2008. https://doi.org/10.1016/j.fct.2008.05.001

Informação da Licença: Este é um artigo Open Access distribuído sob os termos da Licença Creative Commons Attribution, que permite uso irrestrito, distribuição e reprodução em qualquer meio, desde que a obra original seja devidamente citada. 